\title{
Ideación suicida en una muestra de jóvenes víctimas de cyberbullying*
}

\section{Suicidal ideation in one simple of young victims of cyberbullying ${ }^{*}$}

Recibido 01. 09. 2017 • Arbitrado 05. 10. 2017 •

Aprobado 02.11. 2017

Ideación suicida en una muestra de jóvenes víctimas de cyberbullying. Universidad Pontificia Bolivariana. 2016. Investigación institucional.

* Estudiante de pregrado en psicología, Decimo semestre, Universidad Pontificia Bolivariana, diana.larrota.2014@ upb.edu.co

*** Estudiante de pregrado en psicología, Decimo semestre, Universidad Pontificia Bolivariana, raquel. esteban.2013@upb.edu.co

**** Estudiante de pregrado en psicología, Octavo semestre, Universidad Pontificia Bolivariana, yesika.ariza.2013@ upb.edu.co

***** Psicólogo, PhD en Psicología, Docente Universidad Pontificia Bolivariana, jesus.redondo@upb.edu.co

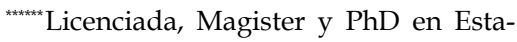
dística, Docente Universidad Pontificia Bolivariana, marianela.luzardo@ upb.edu.co

\section{Resumen}

La presente investigación busca aproximarse a las causas y consecuencias del cyberbullying, respecto a la ideación suicida, en el escenario escolar, caracterizado por ser un fenómeno que afecta el ámbito escolar puesto que se trata de una etapa del ciclo vital donde las cybervíctimas experimentan sentimientos de inferioridad, miedo, ansiedad, estrés e ideas suicidas, entre otras. De este modo, el objetivo de la investigación fue identificar si existen ideas suicidas en jóvenes víctimas de cyberbullying. En este estudio participaron 397 estudiantes de la Institución Nuestra Señora del Pilar de la ciudad de Bucaramanga, con edades comprendidas entre los 10 y 20 años (63.7\% mujeres y $36.3 \%$ hombres), haciendo uso de la escala la Escala de Ideación Suicida de Beck y el Cuestionario de cyberbullying de Ortega, Calmaestra y Mora-Merchán. Así mismo, a través del estudio bibliográfico y los resultados obtenidos de las pruebas se logra evidenciar que el ciberacoso tiene una relación directa con actitudes, intentos y pensamientos suicidas, encontrando que nuestros resultados concuerdan con la literatura, donde se 
afirma que las mujeres son más propensas a sufrir el acoso, mientras que los hombres son más tendentes a ejercerlo.

Palabra claves: Cyberbullying, ideación suicida, bullying, adolescentes, redes sociales.

\section{Abstract}

The present research seeks to approach the causes and consequences of cyberbullying, with respect to suicidal ideation, in the school scene, characterized by being a phenomenon that affects in the school environment since it is a stage of the life cycle where cyber victims experience feelings Of inferiority, fear, anxiety, stress and suicidal ideas, among others. In this way, the objective of the investigation was to identify if there are suicidal ideas in young victims of cyberbullying. This study involved 397 students from the Nuestra Señora del Pilar Institution of the city of Bucaramanga, aged between 10 and 20 years $(63.7 \%$ women and $36.3 \%$ men) using the Beck Suicidal Ideation Scale And the Ortega, Calmaestra and Mora-Merchan cyberbullying questionnaire. Likewise, through the bibliographic study and the results obEstudiante de pregrado en psicología, Universidad Pontificia Bolivariana, 2016.tained from the tests, it is possible to show that cyberactivity has a direct relation with attitudes, attempts and suicidal thoughts, finding that our results agree with the literature, where it is affirmed that women are more Prone to harassment, while men are more inclined to exercise it.

Keywords: Cyberbullying, suicide ideation, bullying, teenagers, social networks

\section{Introducción}

El uso de las tecnologías de información y comunicación (TICs) es un fenómeno de sumo interés que cada día se hace más relevante dentro de la sociedad y en los jóvenes provocando cambios en estos, influyendo en su modo de pensar y de relacionarse (Ramírez, 2015; Ramón, 2015).

En este sentido, el uso de las tecnologías de la información y el fácil acceso a éstas pueden fomentar la vulnerabilidad del adolescente a vivir experiencias, tanto de cyberbullying como de bullying, implicándolos como víctimas, agresores u observadores (Instituto Nacional de Tecnologías de la Comunicación, 2009; Sabater \& López- Hernáez, 2015). Así, el uso de las nuevas tecnologías ha provocado daño y atentado contra la integridad de las víctimas, quienes, en la mayoría de las ocasiones, no pueden defenderse, a pesar de que las TICs no fueron creadas para agredir o maltratar (Ramón, 2015; Redondo, Luzardo e Inglés, 2017; Rial, Gómez, Braña y Varela, 2014; Varela, Pérez, Schwaderer, Astudillo y Lecannelier, 2010). 
Sin embargo, las TICs no son el problema fundamental, pues su buen uso provee al usuario información útil y buenas herramientas que brindan oportunidades de formación, información e investigación. Además, permite una comunicación rápida y fácil con otros usuarios a larga distancia. Por el contrario, el uso inadecuado promueve conductas intimidantes y ofensivas que posibilitan al usuario emitir acciones agresivas y de acoso por las redes sociales bajo el anonimato, siendo esto una ventaja para el agresor. En este sentido, Ramón (2015) defiende la idea de que las personas deben actuar con responsabilidad y respeto en el uso de las mismas, puesto que éstas no fueron creadas con la finalidad de acosar, maltratar, amenazar o agredir a otros, sino con el fin de brindar diversos beneficios a nuestra vida cotidiana, aportando, además, una forma diferente de comunicación e interrelación así como un medio permanente de entretenimiento, diversión y esparcimiento para las personas.

En resumen, el Cyberbullying se trata de un problema que se ha generado a través de internet y las redes sociales, también conocido como intimidación escolar cibernética. Así, Varela et al. (2010) definen el Cyberbullying como "una nueva forma de victimización entre pares que involucra el uso de las tecnologías - teléfono celular, envío de SMS, uso de Internet, etc., para ejercer daño sobre una(s) víctima(s), que no pueden defenderse fácilmente" (p. 348). Otros autores lo explican como las relaciones donde se presentan agresión entre iguales de forma repetitiva y acciones negativas que pueden ser físicas (golpes, heridas con armas, etc.), verbales (insultos, amenazas, etc.) o indirectas (expandir rumores, chismes) (Buelga, Cava y Musitu, 2010; Ramírez, 2015; Ramón, 2015; Redondo, Luzardo y Rangel, 2016).

Cabe señalar que el cyberbullying es un fenómeno muy reciente que nace gracias a la tecnología y se propaga por los medios de comunicación (Buelga et al., 2010; Hernández y Solano, 2007; Ramírez, 2015; Ramón, 2015; Redondo, Luzardo, García e Inglés, 2017; Río, Sádaba y Bringe, 2009), mientras que, a diferencia de éste, el bullying tradicional sólo ocurre en el contexto escolar (Cassiani-Miranda, Gómez-Alhach, Cubides-Munevar y Hernández-Carrillo, 2014). Dicha ventaja de la tecnología permite traspasar los límites de las instituciones educativas hasta los hogares u otros lugares donde aplica la cobertura del internet y emergen las agresiones que traen como consecuencia difamaciones y hostigamientos a la víctima a un nivel mucho más amplio, a veces generando problemas de salud mental como depresión, ansiedad, baja autoestima y, en algunos casos, la ideación suicida (Arenas-Gaitán, Ramírez-Correa y Rondán-Cataluña, 2012; García, 2011; García-Maldonado, Joffre-Velázquez, Martínez-Salazar y Llanes-Castillo, 2011; García-Maldonado et al., 2012; Mendoza, 2012; Redondo et al., 2017). 
Por otro lado, el cyberbullying puede afectar a cualquier persona, sin distinguir edad o género en el uso de las redes sociales, propiciando que los jóvenes estén expuestos a este fenómeno. Además, el cyberbullying presenta características entre las que se podrían destacar el acoso indirecto, formas camufladas de presentarse, el agresor puede ser un desconocido, genera un sentimiento de impotencia y abarca a mayor cantidad de personas, entre otras (Bringué y Sádaba, 2010; Hernández y Solano, 2007; Río et al., 2009).

De igual forma, debido a las características del cyberbullying, se deben considerar los sentimientos de quienes sufren el acoso, el grado de empatía de los agresores o la valoración y preferencia que pueda estar construyéndose por esta nueva forma de maltrato (Avilés, 2009). En este orden de ideas, las víctimas suelen presentar sentimientos como ansiedad, depresión, ideación suicida, estrés, miedo, baja autoestima, nerviosismo e irritabilidad, entre otras, mientras que el victimario puede evidenciar falta de empatía, conductas agresivas y delictivas, bajo autocontrol, impulsividad, entre otras (Garaigordobil, 2011).

Por ello, existe variedad de factores que pueden hacer vulnerable a una persona ante el fenómeno del cyberbullying, como la creación de perfiles por parte de los usuarios, donde, para registrarse, los miembros deben colocar información que desean compartir como los datos sociodemográficos, el nivel de educación, actividades de interés, gustos e información que identifica al usuario, que puede ser aprovechada por otras personas para fines delictivos, abusos, objetos de burla o identificación de posibles víctimas para actividades ilegales (Boldú, 2004; García, 2011; García et al., 2012; Hernández y Solano, 2007).

En cuanto a las diferencias de género y edad, Río et al. (2009) proponen que las mujeres con edades que oscilan entre 15 y 16 años son más propensas a sufrir el acoso, mientras que, por el contrario, los hombres con edades de 14 y 15 años son más propensos a ejercerlo. Así mismo, otra variable donde se evidencian diferencias de género es la relacionada con el abuso en el uso de las redes sociales, es decir, la cantidad de tiempo expuesto a las mismas y el inicio de su uso a muy temprana edad, lo que hace que los jóvenes estén más expuestos a estos riesgos. En este sentido, en un estudio realizado por Varela et al. (2010) se evidencia que los hombres son en mayor frecuencia los victimarios, es decir, ejercen el acoso a sus cybervíctimas, en su mayoría mujeres, las cuales permanecen mayor tiempo en línea.

Del mismo modo, autores como Hezkuntza (2013) plantean que el creciente uso de las redes sociales se da por medio del teléfono celular ya que a cualquier hora del día en periodos cortos o largos pueden hacer uso de éste y 
en cualquier contexto de sus vidas: en la casa, en el colegio, en la calle, etc. Igualmente, aplicaciones como Twitter, Whatsapp, Facebook, Instagram, entre otras, son redes de comunicación que poco a poco han ido abarcando la vida personal y social de los adolescentes.

De esta manera, han aumentado las nuevas problemáticas sociales debido al cyberbullying. Durante mucho tiempo se han tratado de identificar las causas que conllevan a que una persona presente pensamientos suicidas destacando, entre otras, la depresión, las actitudes disfuncionales, bajos niveles de autoestima, la exposición a eventos de vida negativos y la relación de los adolescentes con sus padres (Rial et al., 2014; Sánchez-Sosa, Villarreal-González, Musitu y Martínez, 2011).

Por consiguiente, existen posibles consecuencias del cyberbullying como: alteraciones comportamentales, pérdida de control, sentimientos de culpa, aislamiento, conflictos familiares o descenso en el rendimiento académico, entre otras que podrían llevar a la víctima a deseos o pensamientos suicidas (Avilés, 2009; Carmona, Tobón, Jaramillo y Areiza, 2010; Garaigordobil, 2011; Rial et al., 2014).

En ese sentido, la Organización Mundial de la Salud (OMS) (2003) plantea que más de un millón de personas aproximadamente se suicidan en el año, convirtiendo este fenómeno en un problema de salud pública. Algunos autores afirman que el suicidio en niños y adolescentes es un serio problema mundial que constantemente ha aumentado, siendo la segunda causa de muerte entre las edades de 15 y 29 años (Jaramillo, Rojas, Media y Vallejo, 2015; Mosquera, 2016). Así, Clavijo (2011), en un estudio que realizó entre julio del 2008 y diciembre del 2009 en seis localidades de Bogotá, entrevistando a 482 jóvenes (251 hombres y 231 mujeres), entre los 14 y 17 años de edad, encontró que 94 manifestaron tener ideas suicidas y 69 aseguraron haberlo intentado, algunos de ellos más de una vez.

Así mismo, Areiza (2008) plantea que existen diversas causas que actúan como factor protector o de riesgo frente a la aparición de ideas suicidas, destacando los sentimientos de pérdida como, por ejemplo, la separación de los padres, las decepciones amorosas, el abandono familiar, la sensación de soledad, es decir, lo que se conoce como la "red de apoyo".

$\mathrm{Al}$ respecto, las familias son una de las redes de apoyo que contribuyen a la prevención o rehabilitación de estas personas con ideación suicida (Clavijo, 2011; Montañez, Bartolomé, Montañez y Parra, 2008; Musitu y Cava, 2003). Además, son un sistema que constituye relaciones de intimidad, reciprocidad, dependencia y afecto necesario para formar a cada integrante de 
la misma como un individuo competente con su entorno físico y social con la capacidad de responder a las demandas y exigencias planteadas por el entorno y, así mismo, estimulando estrategias de afrontamiento y toma de decisiones que asegura la supervivencia, sostenimiento, crecimiento y socialización de la familia (Álvarez y Tamayo, 2015; Clavijo, 2011; Estrada-Pineda, Rodríguez-Díaz, Cerros-Rodríguez y Solano-Mendoza, 2015; Montañez et al., 2008; Musitu y Cava, 2003).

Por ello, con esta investigación se pretende identificar la relación existente entre el cyberbullying y la ideación suicida en contextos escolares con el objetivo de ser conscientes de la realidad de este fenómeno para que las instituciones y la sociedad puedan llevar a cabo acciones preventivas a fin de disminuir y evitar la presencia y consecuencias de este fenómeno.

\section{Metodología}

El diseño metodológico de la presente investigación es de tipo descriptivo correlacional y de corte transversal. Según Hernández, Fernández-Collado y Baptista (2006) una investigación de tipo descriptiva correlacional pretende especificar las características o propiedades de individuos, procesos y objetos mediante la medición o recolección de información sobre diversas variables y permite identificar la relación existente entre dos o más variables o categorías, en determinados contextos.

\section{Participantes}

La muestra estuvo constituida por 397 estudiantes de la Institución Nuestra Señora del Pilar de la ciudad de Bucaramanga, con edades comprendidas entre los 10 y 20 años (63.7\% mujeres y 36.3\% hombres). En la Tabla 1 se presenta la frecuencia y porcentaje de los participantes por género y grado.

Para la selección de la muestra, en este estudio se realizó un muestreo aleatorio estratificado según los grados correspondientes desde sexto a once de bachillerato.

Tabla 1: Número (y porcentaje) de sujetos de la muestra clasificados por género y grado

\begin{tabular}{cccc}
\hline \multirow{2}{*}{ Grado } & \multicolumn{2}{c}{ Género } & \multirow{2}{*}{ Total } \\
\cline { 2 - 3 } & Mujer & Hombre & \\
\hline 6 & $76(19.1 \%)$ & $0(0 \%)$ & $76(19.1 \%)$ \\
7 & $24(6 \%)$ & $34(8.6 \%)$ & $58(14.6 \%)$
\end{tabular}




\begin{tabular}{ccccc}
\hline \multirow{2}{*}{ Grado } & \multicolumn{2}{c}{ Género } & \multirow{2}{*}{ Total } \\
\cline { 2 - 4 } & Mujer & Hombre & & \\
\hline 8 & $28(7.1 \%)$ & $40(10.1 \%)$ & 68 & $(17.10 \%)$ \\
9 & $39(9.8 \%)$ & $38(9.6 \%)$ & 77 & $(19.4 \%)$ \\
10 & $47(11.8 \%)$ & $20(5 \%)$ & 67 & $(16.9 \%)$ \\
11 & $39(9.8 \%)$ & $12(3 \%)$ & 51 & $(12.8 \%)$ \\
TOTAL & $253(63.70 \%)$ & $144(36.3 \%)$ & 397 & $(100 \%)$ \\
\hline
\end{tabular}

Nota: por medio de la prueba Chi-cuadrado de independencia entre las variables, se comprueba que existe relación estadísticamente significativa entre las variables grado y género.

\section{$\underline{\text { Instrumentos }}$}

Escala de Ideación Suicida de Beck (ISB). Instrumento que evalúa la recurrencia consciente de pensamientos suicidas (Beck, Rush, Shaw y Emery, 1979), que consta de 19 reactivos aplicados a través de una escala que mide la intensidad de las actitudes, conductas y planes específicos para suicidarse. Cada reactivo se registró en un formato de respuesta de 3 opciones, con un rango de 0 a 2 . Las mediciones se suman para obtener un puntaje global. De este modo, el rango teórico del puntaje es de 0 a 38 .

Hay que destacar que en el caso de que la puntuación relacionada con los ítems (4) "Deseo de 'intentar suicidarse" y (5) "Intento pasivo de suicidarse" es 0 , se suspende la aplicación.

En cuanto a la corrección e interpretación, esta escala consta de tres partes divididas de la siguiente manera: una parte objetiva (circunstancias objetivas relacionadas con la tentativa de suicidio) (ítems 1 a 8), una parte subjetiva: expectativas durante la tentativa de suicidio (ítems 9 a 15) y otros aspectos (ítems 16 a 20). Cuando se interpreta, se le da una valoración de la gravedad de la tentativa (la puntuación total se obtiene sumando las puntuaciones obtenidas en los ítems 1 al 15, señalando que los 5 últimos ítems no puntúan). Así, a mayor puntuación, mayor gravedad (Beck, Schuyler, y Herman, 1974). En esta investigación, el alfa de fiabilidad de Cronbach del instrumento fue de .78 .

Cuestionario de Cibervictimización. Instrumento adaptado del cuestionario diseñado por Smith (2012), y que consta de 27 ítems. Su objetivo es analizar la incidencia o presencia de jóvenes víctimas de cyberbullying. Este cuestionario agrupa siete dimensiones en dos, a saber: episodios de cyberbullying utilizando el teléfono celular y episodios de cyberbullying utilizando Internet. Así mismo, este cuestionario abarca diferentes campos: bullying tradicional, accesibilidad a las TICs (Internet y teléfono celular), cyberbullying a través 
de Internet, y, finalmente, cyberbullying a través del teléfono celular. En este estudio, el alfa de fiabilidad de Cronbach fue de .88 .

\section{Procedimiento}

En primer lugar se solicitó al Rector de la Institución Educativa Nuestra Señora del Pilar el aval para llevar a cabo la investigación. Posteriormente, se entregó el consentimiento informado a los estudiantes, y una vez firmado por los padres o tutores, se inició la aplicación de los cuestionarios desde los grados inferiores hasta los superiores, después de la debida explicación.

La aplicación de las pruebas se llevó a cabo durante una semana y media, en las dos jornadas (mañana y tarde), utilizando aproximadamente una hora. Posteriormente, se realizó la tabulación en Excel y se utilizó el programa IBM SPSS, versión 23, para finalmente analizar los datos.

\section{Resultados}

En la Tabla 2 se muestran los resultados de la relación directa entre las víctimas de cyberbullying, según el género, donde se evidencia que el género femenino es más vulnerable a este tipo de agresión. En este sentido, se puede observar que el $36.8 \%$ de mujeres son víctimas por medio del teléfono celular y el $24.7 \%$ son víctimas a través de internet, a diferencia de los hombres que presentan menores porcentajes frente al rol de víctima a través de estos medios. Además, ser cibervíctima no se relaciona ni con el género ni con los medios por donde se ejerce la agresión ( $\mathrm{p}=.414$ en el caso del teléfono celular $\mathrm{y} \mathrm{p}=.427 \mathrm{si}$ es por internet).

Tabla 2: Victimas de cyberbullying a través del celular e internet

\begin{tabular}{ccccc}
\hline \multicolumn{2}{c}{ Género } & \multicolumn{2}{c}{ Celular } & \multirow{2}{*}{ p } \\
\cline { 1 - 4 } Hombre & Mujer & Total & Chicuadrado & \\
\hline $77(19.4 \%)$ & $146(36.8 \%)$ & $223(56.2 \%)$ & .669 & .414 \\
$50(12.6 \%)$ & $98(24.7 \%)$ & $148(37.3 \%)$ & .632 & .427 \\
\hline
\end{tabular}

Los resultados muestran diferencias significativas entre la ideación suicida en jóvenes cibervíctimas a través del celular $(\mathrm{p}=.05)$.

Respecto a las escalas ISB que prevalecen en las víctimas del fenómeno del cyberbullying por medio del celular, se encontró una relación significativa entre las escalas de razones para pensar o desear un intento suicida $(\mathrm{p}=.023)$, la actitud frente al pensamiento y deseo suicida $(\mathrm{p}=.022)$, el tiempo que gasta 
en el pensamiento o deseo suicida $(\mathrm{p}=.046)$, la realización de un intento de suicidio pasivo $(\mathrm{p}=.011)$, el deseo de intentar un suicidio activo $(\mathrm{p}=.002)$, el deseo de morir $(\mathrm{p}=.011)$, la frecuencia con que se presenta el pensamiento o deseo suicida $(\mathrm{p}=.009)$, el deseo de vivir $(\mathrm{p}=.000)$, razones que detienen el suicidio $(\mathrm{p}=.041)$ y, finalmente, el control sobre la acción y deseo de suicidio $(\mathrm{p}=.000)$ y ser víctima con el celular (Tabla 3$)$.

Tabla 3: Relación entre Ideación suicida y cybervíctimas del celular.

\begin{tabular}{cccc}
\hline Escala ISB & Frecuencia (\%) & Chi Cuadrado & p \\
\hline Deseo de vivir & $17(4.3 \%)$ & 16.456 & .000 \\
Deseo Morir & $20(5.0 \%)$ & 9.074 & .011 \\
Razones para vivir/morir & $19(4.8 \%)$ & 3.893 & .143 \\
Deseo intento suicidio activo & $21(5.3 \%)$ & 12.241 & .002 \\
Realizar intento suicidio pasivo & $25(6.3 \%)$ & 9.106 & .011 \\
Tiempo, pensamiento deseo & $27(6.8 \%)$ & 8.004 & .046 \\
Frecuencia, pensamiento deseo & $20(5 \%)$ & 11.506 & .009 \\
Actitud pensamiento deseo & $32(8.1 \%)$ & 9.594 & .022 \\
Control acción/ deseo suicidio & $11(2.8 \%)$ & 18.433 & .000 \\
Razones detienen el suicidio & $12(3 \%)$ & 8.279 & .041 \\
Razones pensar/ Desear intento suicida & $51(12.8 \%)$ & 9.506 & .023 \\
\hline
\end{tabular}

Finalmente, la Tabla 4 muestra la relación entre la cibervictimización a través de internet y todas las subescalas de la Escala de Ideación Suicida de Beck. Se halló relación significativa entre ser cibervíctima a través de internet y el deseo de vivir $(\mathrm{p}=.000)$, el deseo de morir $(\mathrm{p}=.010)$, razones para vivir / morir $(\mathrm{p}=.002)$, el deseo de realizar un intento suicidio activo $(\mathrm{p}=.003)$, realizar intento suicidio pasivo $(\mathrm{p}=.001)$, tiempo del pensamiento o deseo suici$\mathrm{da}(\mathrm{p}=.010)$, frecuencia con que se presenta el pensamiento o deseo suicida $(\mathrm{p}=.006)$, actitud frente al pensamiento o deseo suicida $(\mathrm{p}=.005)$, control sobre la acción y deseo de suicidio $(\mathrm{p}=.020)$, razones que detiene el suicidio $(\mathrm{p}=.013)$, y razones para pensar o desear el intento suicida $(\mathrm{p}=0.14)$. Además, se evidenciaron asociaciones significativas entre todas las escalas del ISB, por lo que se podría afirmar que en las cibervictimas a través del internet prevalecen y son más frecuentes los deseos, pensamientos e intentos de autolesiones intencionadas. 
Tabla 4: Relación entre Ideación suicida y cybervíctimas de Internet

\begin{tabular}{cccc}
\hline Escala ISB & Frecuencia (\%) & Chi Cuadrado & p \\
\hline Deseo de vivir & $12(3 \%)$ & 15.602 & .000 \\
Deseo Morir & $14(3.5 \%)$ & 9.292 & .010 \\
Razones para vivir/morir & $17(4.3 \%)$ & 12.531 & .002 \\
Deseo intento suicido activo & $16(4 \%)$ & 11.360 & .003 \\
Realizar intento Suicidio Pasivo & $19(4.8 \%)$ & 13.165 & .001 \\
Tiempo, pensamiento deseo & $17(4.3 \%)$ & 11.371 & .010 \\
Frecuencia, pensamiento deseo & $16(4 \%)$ & 12.341 & .006 \\
Actitud pensamiento deseo & $24(6 \%)$ & 13.032 & .005 \\
Control acción/ deseo suicidio & $9(2.3 \%)$ & 9.835 & .020 \\
Razones detienen el suicidio & $7(1.8 \%)$ & 10.756 & .013 \\
Razones pensar/ Desear intento suicida & $18(4.5 \%)$ & 10.677 & .014 \\
\hline
\end{tabular}

\section{Discusión}

El objetivo de esta investigación fue identificar la ideación suicida en jóvenes víctimas de cyberbullying de la Institución Educativa Nuestra Señora del Pilar de Bucaramanga. En primer lugar, los resultados confirman que la cibervictimización se presenta en mayor medida en el género femenino, puesto que se encontró que el $36.8 \%$ de mujeres son víctimas por medio del celular y el $24.7 \%$ a través de internet. Estos resultados concuerdan con lo planteado por diversos autores, quienes afirman que las mujeres son más propensas a sufrir el acoso, mientras que los hombres son más propensos a ejercerlo (Buelga, Cava y Musitu, 2012; Garaigordobil y Alari. 2013; Hezkuntza, 2013; Varela et al., 2010).

Además, también se evidenció que, respecto a las consecuencias de ser víctima de cyberbullying, existe una tendencia de los participantes a tener razones, actitudes, intentos y pensamientos suicidas, reafirmando que la razón para pensar o desear un intento suicida (12.8\%) se relaciona con vivenciar y sufrir este fenómeno. Diversos estudios confirman lo anteriormente dicho al mencionar que algunas de las consecuencias del fenómeno del cyberbullying se presentan en forma de problemas de salud mental como: ansiedad, depresión, estrés, miedo, baja autoestima, nerviosismo, actitudes disfuncionales, exposición a eventos de vida negativos, la relación de los adolescentes con sus padres, las relaciones conflictivas con sus pares, incluso la ideación suicida (Asanza, Flores y Berrones, 2014; Garaigordobil, 2011; Rial at al., 2014; Sánchez-Sosa et al., 2011). 
Finalmente, en cuanto a la victimización por medio del teléfono celular o través del internet, hemos encontrado que existe una correlación significativa entre todas las escalas de ideación suicida y las víctimas a través de internet, lo cual representa un efecto negativo y amenazante de la extensión de las nuevas tecnologías y sus redes para los jóvenes que van hacer uso de ellas con fines humillantes, delictivos, acosadores e intimidadores a otros usuarios que probablemente dichas situaciones los conlleve a pensar, desear autolesionarse intencionalmente como solución ante dicho fenómeno cibernético.

\section{Conclusiones y recomendaciones}

De esta manera, se puede concluir que la etapa de la adolescencia se trata de un periodo de transición donde los jóvenes empiezan a formar y construir su personalidad, un periodo de aprendizaje y socialización con sus pares. Diferentes estudios consideran que la adolescencia es la etapa de transición de la infancia a la vida adulta, donde se pasa de la dependencia a la familia al ingreso a la sociedad con sus derechos y deberes plenos, la cual se estructura desde tres niveles de desarrollo que son: el biológico (con la pubertad), el psicológico (con la responsabilidad) y el social (independencia y valoración social) (Antona, Madrid y Aláez, 2003). Por ende, es de suma importancia el trabajo y el desarrollo de las habilidades sociales, como la empatía, la asertividad, la buena relación con el otro y el fortalecimiento de las redes de apoyo a través de programas como Aulas en Paz propuesto por la Universidad de los Andes (2013) cuyo objetivo es construir una sociedad más pacífica y prevenir la violencia en los colegios, a fin de promover el desarrollo de las habilidades socio-emocionales fundamentales para romper con el ciclo de la violencia.

Por otra parte, se recomienda continuar con el proceso, a través de investigaciones en Colombia acerca del fenómeno del ciberbullying y, a su vez, ampliando los espacios (tiempo, frecuencia y lugares) en los centros educativos para la construcción y adquisición de habilidades o programas de intervención educativa cuyo objetivo sea fomentar conductas protectoras a partir de los factores de riesgo para el posible intento de suicidio, promoviendo herramientas para el abordaje inicial a educadores y padres de familia, logrando así facilitar un excelente desarrollo individual e interpersonal en los adolescentes frente a las problemáticas ocasionadas en el contexto escolar. 


\section{Referencias}

Álvarez, C., Tamayo, P. (2015). Ideaciones suicidas en la adolescencia: lo que debemos saber de las metamorfosis de la pubertad. Revista Poiésis, 30, 79-86. Recuperado de http://www.funlam.edu.co/revistas/index.php/poiesis/article/ viewFile/1849/1474

Antona, A., Madrid, J., Aláez, M. (2003). Adolescencia y salud. Papeles del Psicólogo, 23 (84), 45-53. Recuperado de http://www.papelesdelpsicologo.es/ resumen?pii $=1054$

Areiza, Y. (2008). Posibles causas del intento de suicidio y del suicidio en niños y adolescentes. Revista Electrónica de Psicología Social Poiésis, 1 (15), 1-6. Recuperado de http://www.funlam.edu.co/revistas/index.php/poiesis/article/view/296/285

Arenas-Gaitán, J., Ramírez-Correa, P., Rondán-Cataluña, J. (2012). Uso de los servicios de redes sociales por la generación. Ingeniare: Revista Chilena de ingeniería, 20(3), 425-432. DOI: 10.4067/S0718-33052012000300016

Avilés, J. (2009). Cyberbullying. Diferencias entre el alumnado de secundaria. Boletín de Psicología, No. 96, 79-96, Recuperado de http://www.uv.es/seoane/boletin/ previos/N96-6.pdf

Asanza, M., Flores, E., Berrones, B. (2014). El cyberbullying y sus consecuencias. Recuperado de http://www.eumed.net/rev/cccss/29/cyberbullying.pdf

Beck, A.T., Schuyler, D., Herman, I. (1974). Development of suicidal intent scales. In A.T., Beck, H.L.P., Resnik, \& D.J., Lettieri (eds.), The prediction of suicide, (pp. 45-56). Bowie, MD: Charles Press.

Beck, A.T., Rush, A.J., Shaw, B.F., Emery, G. (1979). Cognitive Therapy of Depression. New York: Guilford Press

Boldú, A. (2014). El ciberacoso: una aproximación criminológica (Tesis de maestría). Universitat Pompeu Fabra, Barcelona. Recuperado de https://repositori.upf.edu/ bitstream/handle/10230/22822/El\%20ciberacoso-TFM.pdf

Bringué, X., \& Sádaba, C. (2010). Niños y adolescentes españoles ante las pantallas: rasgos configuradores de una generación interactiva. CEE Participación Educativa, 15, 86-104. Recuperado de http://dadun.unav.edu/bitstream/10171/18443/1/ n15-sadaba-chalezquer.pdf

Buelga, S., Cava, M., \& Musitu, G. (2010). Cyberbulling: victimización entre adolescentes a través del teléfono móvil de Internet. Psicothema, 22(4), 784-789. Recuperado de http://www.psicothema.com/pdf/3802.pdf 
Buelga, S., Cava, M., Musitu, G. (2012). Validación de la Escala de Victimización entre Adolescentes a través del Teléfono Móvil y de Internet. Revista Panamericana de Salud Pública, 32(1), 36-42. DOI: 10.1590/S1020-49892012000700006

Carmona, J., Tobón, F., Jaramillo, J., Areiza, Y. (2010). Suicidio en la pubertad y la adolescencia. En J. Carmona, F. Tobón, J. Jaramillo, Y. Areiza, Suicidio en la pubertad y la adolescencia (pp.16-18). Medellín: Fundación Universitaria Luís Amigó Fondo Editorial.

Cassiani-Miranda, C., Gómez-Alhach, J., Cubides-Munevar., Hernández-Carrillo. (2014). Prevalencia del bullying y factores relacionados en estudiantes de bachillerato de una Institución Educativa de Cali. Revista Salud pública, 16(1), 14-26. Recuperado de http://www.scielosp.org/pdf/rsap/v16n1/v16n1a02.pdf

Clavijo, G. (2011). Familia poco saludable, una causa de intento de suicidio en Bogotá. Recuperado de http://www.unperiodico.unal.edu.co/dper/article/familia-pocosaludable-una-causa-de-intento-de-suicidio-en-bogota.html.

Estrada-Pineda, C., Rodríguez-Díaz, F. J., Cerros-Rodríguez, E, Solano-Mendoza, C. (2015). Implicaciones parentales en las conductas delictivas de adolescentes: tendencias y narrativas. Papeles de Población, 21(1), 107-132. Recuperado de http://www.redalyc.org/pdf/112/11239488005.pdf

García, M., Martínez, G., González, J., Atenógenes, H., Sánchez, N., Perales, R., Barrientos, M. (2012). Factores de riesgo y consecuencias del cyberbullying en un grupo de adolescentes: Asociación con bullying tradicional. Boletín médico del Hospital Infantil de México, 69(6), 463-474. Recuperado de http://www.scielo. org.mx/scielo.php?script $=$ sci_arttext\&pid $=$ S1665-11462012000600007

García, E. (2011). Redes sociales y cyberbullying. IX encuentro regional de Ampas, Jóvenes y ocio en Extremadura, Miajadas, Abril de 2011.

Garaigordobil, M. (2011). Prevalencia y consecuencias del cyberbullying: una revisión. International Jornal of Psicological Therapy, 11(2), 223-254. Recuperado de http://www.redalyc.org/articulo.oa?id=56019292003

Garaigordobil, M., Alari, J. (2013). Ciberacoso (“Cyberbulling”) en el país Vasco: Diferencias de sexo en víctimas, agresores y observadores. Revista de psicología conductual, 21(3), 461-474. Recuperado de http://online.ucv.es/wp-content/ blogs.dir/15/files/2015/02/Garaigordobil-2013.-Ciberacoso-en-el-Pais-Vasco.pdf

García-Maldonado, G., Joffre-Velázquez, V., Martínez-Salazar, G., Llanes-Castillo, A. (2011). Cyberbullying: Forma virtual de intimidación escolar. Revista Colombiana de Psiquiatría, 40(1), 115-130. DOI: 10.1016/S0034-7450(14)60108-6 
García-Maldonado, G., Martínez-Salazar, G., Saldívar-González, A., Sánchez-Nuncio, R., Martínez-Perales, G., Barrientos-Gómez, M. (2012). Factores de riesgo y consecuencias del cyberbullying en un grupo de adolescentes: Asociación con bullying tradicional. Revista Boletín Médico del Hospital Infantil de México, 69(6), 463-474. Recuperado de http://www.scielo.org.mx/pdf/bmim/v69n6/v69n6a7. pdf

Hernández, R., Fernández-Collado, C., Baptista, P. (2006). Definición y alcance de la investigación a realizar: exploratoria, descriptiva, correlacional o explicativa. Metodología de la investigación. Iztapalapa, México D.F: McGraw-Hill

Hernández, A., Solano, I. (2007). Cyberbullying, un problema de acoso escolar. Revista Iberoamericana de Educación a Distancia, 10(1), 17-36. DOI: http:// dx.doi.org/10.5944/ried.1.10.1011

Hezkuntza, H. (2013). La desigualdad de género y el sexismo en las redes sociales. Vitoria-Gasteiz: ONA Industria Gráfica S.A.

Instituto Nacional de Tecnologías de la Comunicación (2009). Estudio sobre hábitos seguros en el uso de las TIC por niños y adolescentes y confianza de sus padres. León: Autor. Recuperado de http://aui.es/IMG/pdf_estudio_habitos_seguros_ menores_y_econfia nza_padres_versionfinal_accesible_inteco.pdf

Jaramillo, M., Rojas, B., Medina, Ó., Vallejo, C. (2015). Ideación suicida y factores asociados en internos de un establecimiento penitenciario de Antioquia (Colombia). Revista Colombiana de Psiquiatría, 44(2), 100-105. DOI: 10.1016/j. rcp.2015.01.006

Mendoza, E. (2012). Acoso cibernético o cyberbullying: Acoso con la tecnología electrónica. Revista de pediatría de México, 14(3), 133-146. Recuperado de http:// www.medigraphic.com/pdfs/conapeme/pm-2012/pm123g.pdf

Montañez, M., Bartolomé, R., Montañez, J., Parra, M. (2008). Influencia del contexto familiar en las conductas adolescentes. Ensayos, 17, 391-407. Recuperado de https://dialnet.unirioja.es/descarga/articulo/3003557.pdf

Mosquera, L. (2016). Conducta suicida en la infancia: Una revisión crítica. Revista de Psicología Clínica con Niños y Adolescentes, 3(1), 9-18. Recuperado de http://www. revistapcna.com/sites/default/files/mosquera_2016_revision_critica_conducta_ suicida.pdf

Musitu, G., Cava, M. (2003). El rol del apoyo social en el ajuste de los adolescentes. Intervención psicosocial, 12(2), 179-192. Recuperado de https://www.uv.es/lisis/ mjesus/8cava.pdf 
Organización Mundial de la Salud (OMS) (2003). Informe sobre la salud en el mundo 2003. Recuperado de http://www.who.int/whr/2003/en/whr03_es.pdf

Ramírez, M. (2015). Bullying y Cyberbullying entre adolescentes de Educación Secundaria. (Tesis de grado). Universitas Miguel Hernández, España. Recuperado de http://dspace.umh.es/bitstream/11000/2597/1/Ramirez\%20Cuevas_Marina. pdf

Ramón, T. (2015). Ciberbuylling entre jóvenes. (Tesis de grado). Universidad de la Laguna, España. Recuperado de https://riull.ull.es/xmlui/bitstream/ handle/915/1826/CIBERBULLYING\%20ENTRE\%20LOS\%20JOVENES\%20. pdf?sequence $=1$

Redondo, J., Luzardo, M., Rangel, K. (2016). Ciberagresión: un estudio sobre la prevalencia en estudiantes universitarios colombianos. Informes Psicológicos, 16(1), 85-99. Recuperado de https://revistas.upb.edu.co/index.php/ informespsicologicos/article/view/6482

Redondo, J., Luzardo, M., Inglés, C.J. (2017). Prevalencia de la ciber victimización en estudiantes de la Universidad Pontificia Bolivariana de Bucaramanga. Revista Criterios, 23(1), 307-322. Recuperado de http://www.umariana.edu.co/ojseditorial/index.php/criterios/article/view/1202

Redondo, J., Luzardo, M., García, K. L., Inglés, C. J. (2017). Impacto psicológico del ciberbullying en estudiantes de la Universidad Pontificia Bolivariana, seccional Bucaramanga: un estudio exploratorio. Revista Colombiana de Ciencias Sociales, 8(2), 458-478. DOI: http://dx.doi.org/10.21501/

Rial, A., Gómez, P., Braña, T., Varela, J. (2014). Actitudes, percepciones y uso de internet y las redes sociales entre los adolescentes de la comunidad gallega (España). Anales de Psicología, 30(2), 642-653. DOI: 10.6018/analesps.30.2.159111

Río, J., Sádaba, C., Bringe, X. (2009). Menores y redes ¿sociales?: de la amistad al cyberbullying. Revista de Estudios de juventud, 10(88), 115-129. Recuperado de http://www.injuve.es/sites/default/files/RJ88-09.pdf

Sabater, C., López-Hernáez, L. (2015). Factores de riesgo en el cyberbullying. Frecuencia y exposición de los datos personales en Internet. International Journal of Sociology of Education, 4(1), 1-25. DOI: 10.4471/rise.2015.01

Sánchez-Sosa, J.C. Villarreal-González. M., Musitu, G., Martínez, B. (2011). Ideación Suicida en Adolescentes: Un Modelo Explicativo. En Sánchez-Sosa, J.C. y Villarreal-González, M. (Eds.), Tópicos de Psicología de la Salud en el Ámbito Universitario (15-34). Monterrey: Universidad Autónoma de Nuevo León Consorcio de Universidades Mexicanas 
Smith, P.K. (2012). Cyberbullying and cyber aggression. En Shane R. Jimerson, Amanda B. Nickerson, Matthew J. Mayer, \& Michael J. Furlong (Eds.), Handbook of School Violence and School Safety: International Research and Practice (pp.93104). New York, NY: Routledge.

Universidad de los Andes (2013). Aulas en Paz. Recuperado de https://aulasenpaz. uniandes.edu.co/

Varela, T., Pérez, C., Schwaderer, H., Astudillo, J., Lecannelier, F. (2010). Caracterización de cyberbullying en el gran Santiago de Chile, en el año 2010. Revista Quadrimestral da Associação Brasileira de Psicologia Escolar e Educacional, 18(2), 347- 354. DOI: 10.1590/2175-3539/2014/0182794 